\title{
RESEÑA: SEXO Y GÉNERO EN MEDICINA. UNA \\ INTRODUCCIÓN A LOS ESTUDIOS DE LAS MUJERES Y \\ GÉNERO EN LAS CIENCIAS DE LA SALUD. MONTSERRAT \\ CABRÉ I PARET Y FERNANDO SALMÓN MUÑIZ ${ }^{1}$
}

\author{
Yamila Azize Vargas ${ }^{2}$
}

\begin{abstract}
Montserrat Cabré i Paret y Fernando Salmón Muñiz (eds.), Sexo y género en medicina. Una introducción a los estudios de las mujeres y género en las ciencias de la salud. Santander: Ediciones Universidad de Cantabria, 2013. ISBN: 978-84-8102675-7.
\end{abstract}

Los esfuerzos para introducir un enfoque feminista en la enseñanza de la medicina, siguen abriéndose paso en la academia, gracias, en su inmensa mayoría a la gestión de mujeres en las ciencias. Desde fines del pasado siglo, durante los años 1990, comenzaron a trabajarse estos espacios, favorecidos por el surgimiento de los estudios de las mujeres y de nuevas investigaciones científicas con perspectivas críticas sobre una variedad de temáticas que documentaban con nitidez la construcción de estereotipos y visiones sexistas en las ciencias en la teoría y práctica científicas. Entre los múltiples retos que confrontan estas iniciativas, particularmente en las ciencias de la salud, es la necesidad de libros de texto que reúnan investigaciones sólidas, con perspectiva interdisciplinaria y poder de persuasión para un campo, como el científico y en particular, el de la medicina, que persigue "descubrir una verdad" para resolver problemas, la llamada "objetividad científica" aislada frecuentemente de cualquier contexto cultural o político.

\footnotetext{
1 Fecha de recepción: 24/07/2014.

Fecha de aceptación: 07/08/2014.

2 Directora del "Programa Salud Pro Mujer", Departamento de Obstetricia y Ginecología, Escuela de Medicina, Universidad de Puerto Rico, Puerto Rico,EE.UU; $₫$ yazizevargas@gmail.com.
} 
Dentro de este panorama, la publicación del libro Sexo y género en medicina: es una aportación importante para aquella docencia interesada, en según dicen los editores, promover "una reflexión que es fundamental para garantizar un análisis serio de nuestro sistema de salud y para configurar una actitud responsable y crítica....". ${ }^{1}$ El libro surge como parte de una iniciativa docente en la Escuela de Medicina de la Universidad de Cantabria dirigida, "a quienes van a integrarse como profesionales en el sistema sanitario". Tiene un enfoque interdisciplinario con temáticas sobre biología, investigación clínica, historia, práctica sanitaria, política feminista. La mayoría de las investigaciones incluidas, cuestionan desde diferentes ángulos el pensamiento tradicional que sostiene el sexismo y la subordinación de las mujeres en la teoría científica y práctica clínica.

La experiencia de más de una década en Puerto Rico con el curso Mujer y salud, un curso muy afín al que inspiró este libro, es un ejemplo que confirma la tesis que propone el libro Sexo y género en medicina... de que sólo con investigaciones sólidas “...podrán romperse las rutinas que impiden las transformaciones sociales" (énfasis mío). Mis estudiantes consistentemente expresaron en sus evaluaciones del curso cómo el enfoque y contenido, las lecturas utilizadas, "rompieron los esquemas tradicionales de análisis...", les ayudaba a pensar críticamente, les "permitió abrir horizontes y exponerse a nuevas maneras de pensar ante temas conflictivos y que retan nuestros principios y tradiciones. ${ }^{2}$ En tan sólo dos semanas, el curso era capaz de cuestionar paradigmas inculcados por la educación médica tradicional y visibilizar temáticas censuradas sobre la salud de la mujer. El impacto fue tan notable que provocó hasta una investigación que, aunque no era su intención, contribuyó a la discusión de políticas educativas sobre salud sexual reproductiva en la Escuela de Medicina y a documentar la valoración favorable hecha por el estudiantado. ${ }^{3}$

Tres grandes temas se desarrollan en el libro: I. Sexismo en la educación médica y práctica clínica II. Historias misóginas en la medicina III. Protagonismo de las mujeres

\footnotetext{
${ }^{1}$ El libro se puede comprar a través del website de la Editorial de la Universidad de Cantabria http://www.editorial.unican.es/libro/sexo-y-genero-en-medicina.

${ }^{2}$ Ver Evaluaciones Curso Mujer y Salud en http: www.saludpromujer.md.rcm.upr.edu.

${ }^{3}$ Resolución Senado Académico, 2006; Ponencia de la Dra. Juana Rivera, Directora Departamento de Obstetricia y Ginecología, ante el Comité Investigador del Senado Académico del Recinto de Ciencias Médicas.
} 
en el sector salud. En el primer tema dos artículos (E. Martin, Lawrence y Bendixen) abordan el tema del sexismo en libros de texto utilizados en la educación médica y otro artículo, de S. Kessler, ilustra un caso en práctica clínica. El de Martin discute la construcción de estereotipos sexistas en textos educativos por medio de descripciones humanizadas del funcionamiento del óvulo ("derrocha células germinales, es pasivo y tiene que ser rescatado") y de los espermatozoides ("producidos por millones y aunque pequeños, viajan y son activos y aerodinámicos"). El otro caso de sexismo en los libros de texto, documenta con ejemplos de 31 libros utilizados a lo largo de 100 años, que el principio rector de su análisis es que "la anatomía del hombre es lo que establece la norma, y la de la mujer es la que se desvía de esa norma....los cuerpos femeninos se presentan como variantes de los cuerpos masculinos". Estos dos estudios de caso, son sin duda un buen trasfondo para ver cómo estas visiones androcéntricas operan en prácticas médicas como el manejo clínico de la intersexualidad, tema del tercer artículo. A través de entrevistas a médicos especialistas de casos de infantes intersexuales, se ilustra cómo las preconcepciones de género influyen en las intervenciones clínicas para asignar el sexo. La gran ironía que documenta la investigación es que, a pesar de los avances tecnológicos y quirúrgicos para "descubrir, diagnosticar y construir el verdadero sexo del bebé intersexual", la decisión final de asignación del sexo está determinada mayormente por ideas y estereotipos culturales.

El segundo tema, historias misóginas en la medicina, se elabora con dos artículos que discuten temas de salud sexual reproductiva (menstruación, control de la fertilidad) y un tercero que documenta la exclusión sistemática de las mujeres en investigaciones clínicas. En torno a la menstruación, el artículo de Cabré I Paret y Salmón Muñiz, hace historia de la trayectoria de la estigmatización de esta función fisiológica en el ámbito médico y religioso del Siglo XVI y las repercusiones negativas en la vida cotidiana de las mujeres de aquella época. En este período los círculos médicos universitarios atribuían la "responsabilidad" a las mujeres y sus menstruaciones como las causantes del mal de ojo. Los autores concluyen que "la explicación médica del mal de ojo contribuyó a la tendencia de la supresión de lo femenino en los discursos culturales dominantes del Siglo 
XVI". Discurso que todavía persiste en el lenguaje coloquial con frases como "estoy mala" y en prácticas punitivas de algunos cultos religiosos.

El otro artículo sobre salud sexual reproductiva de Lara Marks aborda un tema central en la historia de la salud de las mujeres -la experimentación clínica con mujeres sanas para elaborar los anticonceptivos orales que planificada en Estados Unidos, se ensaya en Puerto Rico. Sin embargo, el análisis elaborado por la autora se queda corto en contextualizar políticamente y con una perspectiva feminista las fuentes originales que utiliza. El artículo se propone destacar "las complejidades que conllevaron aplicar al cuerpo humano un modelo molecular reduccionista en medicina": el reclutamiento y retención de mujeres en el estudio, la selección del personal y las estrategias utilizadas para obtener resultados convincentes del uso de la píldora. La correspondencia de los investigadores del ensayo, una de las fuentes principales del artículo, pone de manifiesto las visiones racistas, sexistas y colonialistas del proyecto que olvidó el ideario feminista de control de la fertilidad para ponerse al servicio de políticas de control poblacional. Expresiones como la necesidad de una "jaula de mujeres ovuladoras", o la queja de la "falta de cooperación activa de las mujeres", las estrategias de reclutar mujeres analfabetas funcionales o totales, mujeres esquizofrénicas o mujeres confinadas, pone en evidencia esas visiones. Los problemas de retención a causa de los efectos secundarios son vistos por la autora como uno de los "obstáculos" confrontados, y no como una muestra de resistencia/protesta por parte de las mujeres. Las declaraciones del investigador principal del proyecto en el sentido de que los efectos secundarios "más que reacciones reales, se trataba de síntomas psicosomáticos", se citan pero no se cualifican (Marks 2013: 191), como tampoco se valoran las advertencias hechas sobre estos efectos secundarios por dos mujeres médicas investigadoras en el proyecto. ${ }^{4}$ El tiempo daría la razón a estas médicas, a las mujeres desertoras, a las que "no cooperaron activamente", y a cientos de mujeres que, una vez que el anticonceptivo estuvo en el mercado, denunciaron los efectos secundarios y muertes adicionales a causa de los anticonceptivos.

\footnotetext{
${ }^{4}$ Lara Marks (2013: 192) da como referencia una entrevista que hizo a la Dra. Satterthwaite en 1995 y también cita a Annette Ramírez de Arellano y Conrad Seipp 1983: 120-121. La Dra. Edris Rice-Wray advertía sobre la posibilidad del fracaso del proyecto debido a los efectos secundarios de la pastilla ensayada. Posteriormente, la Dra. A.P. Satterthwaite tuvo tensiones con Pincus porque consideró que el anuncio de que la píldora inhibía el cáncer de cuello uterino era muy prematuro.
} 
El ensayo en Puerto Rico registró tres muertes que nunca fueron investigadas, algo que no menciona el artículo, y posteriormente en Estados Unidos se registraron otras 11 muertes de mujeres (Ramírez de Arellano y Seipp 1984: 122). El artículo menciona, pero no discute, el hecho de que, según cita, el cálculo de participantes se hizo por ciclos menstruales en vez de por número de mujeres para transmitir la idea de que habían logrado un anticonceptivo seguro y fiable (Marks 2013: 192). Una corrección pertinente es que, contrario a lo que dice el artículo, las leyes Comstock, que prohibían este tipo de proyecto en Estados Unidos, sí estuvieron vigentes en Puerto Rico, ${ }^{5}$ pero fueron ignoradas tan temprano como en 1938 para dar paso a uno de los primeros ensayos de esterilización masiva en el mundo auspiciado por Estados Unidos. Para el 1956, fecha del ensayo de la píldora, ya existía una red de clínicas y personal adiestrado en el campo del control de la natalidad. Puerto Rico, colonia de Estados Unidos, constituía un lugar excepcional para volver a ensayar políticas de control poblacional como la de la píldora anticonceptiva. ${ }^{6}$

Esta historia ejemplifica el argumento del último artículo de este tema, de Sue Rosser, sobre el sesgo androcéntrico en la investigación clínica en el sentido de que las mujeres han sido incluidas en la experimentación para controlar la reproducción, pero sistemáticamente excluidas en otras áreas de investigación clínica. El artículo hace un recuento de casos de investigaciones en Estados Unidos en que se excluyen a las mujeres de ensayos clínicos sobre medicamentos para colesterol, afecciones coronarias, financiamiento de proyectos. Como ejemplo de una investigación clínica que sí atiende las necesidades de las mujeres -y que recién comenzaba para la fecha en que se escribe el artículo- se menciona el estudio Women's Health Initiative (WHI), el más abarcador sobre salud de las mujeres hecho en Estados Unidos auspiciado por el National Institute of Health, y que tendría implicaciones importantísimas al vincular las terapias hormonales con el cáncer del seno y ataques del corazón en Estados Unidos. Los resultados del

\footnotetext{
${ }^{5}$ En 1938, a raíz de varios proyectos de ley aprobados por la legislatura de Puerto Rico para hacer posible el proyecto de esterilización, la Iglesia Católica impugnó dichas leyes argumentando la vigencia de las leyes Comstock y llevó un caso a la Corte de Distrito de Estados Unidos que reconoció la vigencia de las leyes Comstock en Puerto Rico. Sin embargo, en la práctica, las prohibiciones de las leyes Comstock no fueron respetadas (Ramírez de Arellano y Seipp 1984: 51-56).

${ }^{6}$ La operación, Dirección y Producción: Ana María García, V.O. inglés, con entrevistas en español, color, DVD\&VHS, 40 min., 1982; The Pill, Dirección: Erna Buffie and Elise Swerhone. Producción: National Film Board of Canada V.O. inglés, color, V.H.S., 45 min, 1999, Canadá.
} 
estudio (2002) provocaron que casi la mitad de mujeres en estas terapias abandonaran su uso, con la consecuencia de una reducción significativa en la incidencia de cáncer del seno (Rasanagayam 2012). El estudio del WHI, es un buen ejemplo de lo que sucede cuando se supera el "sesgo androcéntrico" y se incluyen temas pertinentes de la salud de las mujeres.

El tercer tema, el del protagonismo de las mujeres en el sector salud, se aborda desde tres diferentes sectores: el informal, el institucional y el político. El sector informal, en el artículo de Rosa María Osorio que estudia el manejo clínico efectivo de enfermedades respiratorias por medio de la auto atención, diagnóstico y automedicación que hacen un grupo de madres mexicanas con sus infantes. La investigación sugiere la importancia de que el sector sanitario tome en cuenta estos saberes populares del sector informal a fin de instrumentar políticas que los recuperen en beneficio de la salud de la población. Estas recomendaciones recuerdan el fenómeno contemporáneo y transnacional del misoprostol para la auto inducción de aborto. Lo que comenzó como una práctica clandestina entre las mujeres para abortar en la década de 1980, en contextos donde el aborto está criminalizado o no está accesible, se ha convertido en una práctica avalada por organizaciones de salud institucionales dada la reducción en las complicaciones y muertes por abortos inseguros en que ha resultado su uso ${ }^{7}$.

El segundo grupo de mujeres, las médicas, abordado por el artículo de Delgado, Távora y Ortiz estudia "los discursos que un grupo de jóvenes médicas españolas construye acerca de su experiencia profesional". Los hallazgos sugieren que persiste una visión esencialista para explicar diferencias entre mujeres y hombres, algo que no contribuye a enriquecer su valoración profesional y pone de manifiesto un conflicto de pertenencia entre vida familiar y profesional y que problematiza su desempeño en el campo de la medicina. Polémicas recientes sobre el "problema de la feminización de la medicina" en España, ponen en evidencia que las dificultades y retos que todavía enfrentan las mujeres en la medicina. ${ }^{8}$

\footnotetext{
${ }^{7}$ World Health Organization, Federación Internacional de Ginecología y Obstetricia,

${ }^{8} \mathrm{La}$ polémica se generó a raíz de un artículo de Alicia Serrano publicado en El Diario Médico el 18 de junio del 2012 titulado La crisis agudiza los problemas de la feminización. A esto siguió una carta de Maite
} 
El último grupo de mujeres, el de las activistas, se aborda en el ensayo escrito por Kathy Davis que relata el proceso de traducción a más de 20 lenguas entre las organizaciones de mujeres que participaron en el proyecto de adaptación del libro Nuestros cuerpos, nuestras vidas publicado en el año 1971 por el Colectivo de Mujeres de Boston. Este parte de una crítica a la medicina institucionalizada con el propósito de brindar información y recursos a las mujeres. Davis argumenta que el proyecto fue "un buen ejemplo de las posibilidades y de las limitaciones del feminismo global", dado sobre todo por el respeto, flexibilidad y aprendizaje que reflejó el proceso.

\section{Comentarios finales}

Mi entusiasmo sobre Sexo y género en medicina está fundamentado por la puesta en práctica que hemos hecho de partes del contenido y el enfoque del libro en la Escuela de Medicina de Puerto Rico. De igual modo, el libro tiene la posibilidad de ser utilizado en otros en departamentos de medicina y ciencias de la salud tanto en España como en América Latina y el Caribe, donde ya existen valiosas iniciativas docentes sobre mujer y salud. ${ }^{9}$ El libro será sin duda un recurso educativo muy valioso para todas estas iniciativas.

Para futuras ediciones, sería útil añadir comentarios que actualicen discusiones como las del manejo clínico de la intersexualidad, en específico los cuestionamientos a las teorías de Money; la participación de mujeres en ensayos clínicos a la luz de los resultados del Women's Health Initiative; e incorporar visiones feministas y políticas a la

Paramio Nieto, Presidenta de la Asociación de Mujeres Investigadoras y Tecnólogas (AMIT) de Cataluña titulada Feminización de la medicina publicada en El País, el 6 de julio 2012 y dos artículos adicionales publicados en El País el 13 de julio del 2012: El aluvión de médicas encuentra recelos por Jaime Pratts y Doctoras, no; enfermeras sí, de Gabriela Casañas.

${ }^{9}$ En España destacan Teresa Ortiz de la Universidad de Granada (ver su libro Medicina, historia y género), Consuelo Miqueo de la Universidad de Zaragoza y Pilar Sánchez López de la Universidad Complutense; en América Latina y el Caribe sobresale la investigación de Dora Cardacci que hace un inventario y análisis sobre los programas e iniciativas existentes en las Facultades de Medicina de la región en Perspectiva de género y derechos reproductivos en curricula de medicina latinoamericanos. 
historia del ensayo de la píldora anticonceptiva. Una ausencia importante es la del tema del aborto. La educación y sensibilización sobre el aborto como un asunto de salud es tarea prioritaria en la práctica docente de las ciencias médicas. En el caso del los países donde se habla español, a quienes está destinado este libro, la población de mujeres hispanoparlantes es uno de los grupos más numerosos y a riesgo por la criminalización del derecho al aborto y dificultad de acceso a servicios. En el caso de América Latina y el Caribe, según la Organización Mundial de la Salud, la región tiene la tasa más alta del mundo de aborto inseguro. En España, la amenaza de restricciones legales ha puesto a riesgo el acceso al aborto para la mayoría de las mujeres. En nuestra experiencia docente, el estudiantado identificó el aborto como uno de temas de más relevancia del curso. Sin embargo, el aborto en la academia y en la formación de profesionales de la salud continúa siendo un tema del que no se habla. ${ }^{10}$

Una de las lecciones en el campo de la salud de las mujeres, y este libro ofrece varios ejemplos, es el reconocimiento de la contribución para generar el cambio que han hecho investigadoras y activistas feministas. Esto constituye un referente a tener en cuenta, especialmente para los dos sectores en la academia, los facultativos, y el estudiantado, quienes serán los responsables de generar aquellas transformaciones curriculares que posibilitarán mejores prácticas sanitarias. Esas tareas curriculares tienen en el libro Sexo y género en medicina un instrumento idóneo para ayudar en la tarea de romper las rutinas sexistas en las ciencias y así contribuir a cultivar el respeto a las mujeres y promover una mejor medicina.

\footnotetext{
${ }^{10}$ Entre el año 1997 hasta el año 2010 Saludpromujer realizó doce encuestas diferentes a diversos grupos de estudiantes en las ciencias médicas y profesionales de la salud. Como estrategia educativa para fomentar la formación de estos sectores y del público, se hicieron una serie de programas radiales - De eso no se habla- que luego se produjeron en CD's. Toda la serie se encuentra en www.saludpromujer.md.rcm.upr.edu
} 


\section{Referencias bibliográficas}

Marks, Lara. 2013. "Una jaula de mujeres ovuladoras. Historia de los primeros ensayos clínicos de la píldora oral anticonceptiva, 1950-1959”. Sexo y Género en Medicina. Eds. Montserrat Cabré I Paret y Fernando Salmón Muñiz. Santander: Ediciones Universidad de Cantabria. 165-198.

Ramírez de Arellano, Annette y Conrad Seipp. 1984. Colonialism, Catholicism and Contraception. A History of Birth Control in Puerto Rico. Chapell Hill: The University of North Carolina Press.

Rasanagayam, Sharima. 2012. Reducing breast cancer risk: what we've learned from the Women's Health Initiative. Breast Cancer Fund. July 10, 2012. http://www.preventionstartshere.org/reducing-breast-cancer-risk-what-wevelearned-from-the-womens-health-initiative/ [11 jul. 2012]

Ortiz, Teresa. 2006. Medicina, historia y género. Oviedo: KRK.

Cardacci, Dora. 2002. Perspectiva de género y derechos reproductivos en curricula de medicina latinoamericanos. México. UNPFA. 